\title{
论文
}

\section{基于TIMED/SABER卫星温度数据对大气经验模 型的评估}

程旋 ${ }^{1,2}$, 肖存英 ${ }^{1 *}$, 胡雄 $^{1}$, 杨钧烽 ${ }^{1}$

1. 中国科学院国家空间科学中心临近空间环境研究室, 北京 100190 ;

2. 中国科学院大学, 北京 100049

*联系人, E-mail: xiaocy@nssc.ac.cn

收稿日期：2018-03-30; 接受日期：2018-04-04; 网络出版日期：2018-07-31

国家重点研究发展计划(编号: 2016YFB0501503)和高分青年基金项目(编号: GFZX04061502)资助项目

摘要 以TIMED/SABER观测的2002-2016年共计 15 年的全球温度数据为标准, 与USSA-76及NRLMSISE-00模式 温度数据对比分析. 通过计算大气模式与卫星观测的温度偏差、温度偏差的标准差及均方根误差, 分析两种大气 模型的适用性. 通过设置模式与卫星观测温度偏差, 计算不同纬度地区温度偏差要求下模式的置信度. 结果表明: (1) 两个大气模型计算的温度数据与卫星观测的温度数据随高度的变化具有较好的一致性; (2) NRLMSISE-00模 式的日平均和月平均精度高于USSA-76模式; (3) 两个大气模式在冬夏季高纬地区及中纬地区UMLT高度表征真 实大气的能力有待提高, NRLMSISE-00模式表征真实大气的能力整体优于USSA-76模式; (4) 同一模式在相同纬 度地区和温度偏差要求下, 置信度随着高度的升高而降低; 相同的高度和温度偏差要求下, 置信度随着纬度的升 高而降低. 通过分析USSA-76和NRLMSISE-00模式与卫星观测数据之间的差异, 对大气模式在工程中的应用提供 参考, 为大气模式的后续修正提供依据.

关键词大气模型, 温度, TIMED/SABER, NRLMSISE-00, USSA-76, 评估

PACS: 43.28.Vd, 46.15.-x, 96.12.Jt, 92.60.hv

\section{1 引言}

近年来, 临近空间飞行器发展迅猛, 对临近空间大 气环境提出了迫切需求. 大气温度是临近空间重要的 大气参量之一, 也是飞行器设计阶段重要的基本输入 参量之一. 大气温度对飞行器推进系统的关键参数有 较大的影响，如推力、耗油率等 ${ }^{[1,2]}$. 大气模式是提供
临近空间大气温度的重要途径，模式温度对实际大气 温度的表征能力是大气模式选择的重要衡量标准. 基 于观测数据对大气模式进行评估及适用性分析，可为 飞行器气动设计、航迹规划、控制设计、建模仿真等 工程应用提供参考，同时对大气模式的后续修正有重 要的参考价值.

临近空间大气参量的探测主要基于地面雷达和卫

引用格式: 程旋, 肖存英, 胡雄, 等. 基于TIMED/SABER卫星温度数据对大气经验模型的评估. 中国科学: 物理学 力学 天文学, 2018, 48: 104701 Cheng X, Xiao C Y, Hu X, et al. Evaluation of atmospheric empirical model based on TIMED/SABER satellite temperature data (in Chinese). Sci SinPhys Mech Astron, 2018, 48: 104701, doi: 10.1360/SSPMA2018-00091 
星的遥测、探空气球和探空火箭的原位探测. 这些探 测技术的发展为大气模式的开发和改进提供了重要的 数据支持, 建立起了在航空航天领域应用较为广泛的 标准大气模型和参考大气模型. 其中具有代表性的标 准大气模型有USSA-62和USSA-76, 采用了大量的火 箭探测数据作为数据源 ${ }^{[3]}$. 其中USSA-76模式能够表 征中等太阳活动条件下中纬度区域0-1000 km高度理 想化稳态大气的平均状态. 具有代表性的参考大气模 型有GRAM系列模式 ${ }^{[4]}$ 、MSIS系列模式 ${ }^{[5,6]}$ 等. 其中 NRLMSISE-00大气模型是利用探空火箭、卫星质谱 仪和地面非相干散射雷达等观测资料建立起来的，能 够提供全球不同地理经纬度、季节下从地面到逃逸层 高度大气的温度、密度等参量, 广泛应用于科学研究 和工程领域 ${ }^{[7-10]}$.

标准大气模型和参考大气模型是利用大气理论和 大气探测数据建立起来的经验模型, 与实际大气相比, 存在一定的误差. 国内外学者对大气模型开展了一系 列的评估. 陈亮等人 ${ }^{[9]}$ 通过计算NSPM(Near Space Parameter Model)模式与激光雷达数据之间的平均精 度、平均残差和标准差，评估了NSPM模式的适用性. 汪宏波等人 ${ }^{[11]}$ 采用CHAMP卫星的加速仪数据反演的 大气密度评估了太阳活动峰年时DTM-94和MSIS-90 模型的精度. 万田等人 ${ }^{[12]}$ 通过对比TIME/SABER探测 器观测的大气密度数据和地基Rayleigh激光雷达观测 的大气密度数据, 计算特定误差带下大气密度的置信 度的方法，计算了WACCM大气数值模型、USSA-76 和NRLMSISE-00大气经验模型在 80,90 和 $100 \mathrm{~km}$ 的误 差带和置信度, 结果表明, 3 种模式满足高置信度下的 误差带不能满足飞行器气动设计的需求. 徐寄遥等 人 $^{[13]}$ 通过计算TIME/SABER探测器与NRLMSISE-00 大气模型月平均全球温度分布，对比发现中间层和低 热层区域的卫星温度数据与大气模型计算结果存在较 大的差异.

与万田等人 ${ }^{[12]}$ 对 $100 \mathrm{~km}$ 附近大气模式密度的评估 方法类似, 本文针对临近空间 20-100 km大气模式温度 进行评估, 基于TIMED卫星上的SABER探测器探测的 2002-2016年共计 15年的全球大气温度数据, 采用数学 统计的方法对USSA-76和NRLMSISE-00模式温度在 临近空间20-100 km高度范围下的适用性进行评估, 包 括模式日平均和月平均温度数据的评估. 文中统计分 析了 15 年不同纬度区域不同温度偏差要求下的样本
数, 计算偏差约束下的置信度, 分析USSA-76和 NRLMSISE-00大气模型在不同纬度区域的适用性. 通 过分析USSA-76和NRLMSISE-00模式与卫星观测数 据之间的差异，对大气模式在工程中的应用提供参考， 同时有助于大气模式的后续修正.

\section{2 数据来源和评估分析方法}

\section{1 数据来源}

2001年12月7日上午7:07, 美国宇航局在加利福尼 亚州范登堡空军基地成功发射了 TIMED (Thermosphere Ionosphere Mesosphere Energetics and Dynamics)卫星, 轨道倾角为 $74.1 \pm 0.1^{\circ}$, 轨道高度为 $(625 \pm 25) \mathrm{km}$, 卫星围绕地球的周期约 $1.6 \mathrm{~h}$. TIMED卫 星搭载的探测仪器SABER (Sounding of the Atmosphere Using Broadband Emission Radiometry)是一个 10 通道红外辐射计, 在卫星飞行过程中采用临边扫描 的方式对大气中 $\mathrm{CO}_{2}$ 的辐射信号进行探测, 进而反演 出高度方向上的大气温度、密度、气压等大气参量的 垂直廓线. SABER探测器的垂直分辨率约为 $2 \mathrm{~km}^{[14]}$, 卫星缓慢进动，观测数据覆盖全球 $24 \mathrm{~h}$ 地方时的周期 约为 $60 \mathrm{~d}^{[15]}$.

TIMED/SABER探测器自2002年以来, 为科学研 究提供了大量连续可靠的实测数据. 其温度数据的可 靠性已得到验证 ${ }^{[16-18]}$. Wrasse等人 ${ }^{[19]}$ 将CHAMP卫星的 温度数据和TIMED/SABER的温度数据做了对比, 两 个卫星采用了完全不同的探测温度的方法，在 15-35 km 高度温度的平均偏差约为 $2 \mathrm{~K}$, 标准偏差小 于 $3 \mathrm{~K}$; 在中低纬度 $\left(-30^{\circ} \mathrm{N}-30^{\circ} \mathrm{N}\right)$, 温度偏差从 $15 \mathrm{~km}$ 处的 $0-1 \mathrm{~K}$ 增加到 $35 \mathrm{~km}$ 处的 $-4 \mathrm{~K}$. 宫晓艳等人 ${ }^{[20]}$ 将 COSMIC大气掩星的温度数据与 TIMED/SABER的温 度数据进行了比较, 两种温度数据得出了趋势一致的 温度垂直廓线; 在 $38 \mathrm{~km}$ 平均温度偏差在 $0 \mathrm{~K}$ 左右, $38 \mathrm{~km}$ 以上, 温度偏差随高度增加, 最大值约为 $5 \mathrm{~K}$, 数 据相对吻合较好. 此外SABER数据在大气参数的日变 化、季节变化、年变化、纬度变化、高度变化及大气 扰动等大气科学研究中已得到了广泛的应用 ${ }^{[21-26]}$. 文 献[19]给出了SABER探测器精度的分析结果, 可忽略 多年统计时仪器的测量误差以及数据处理时的抽样误 差. 因此, 本文忽略SABER数据的测量误差, 以该数据 为衡量标准, 统计评估USSA-76和NRLMSISE-00大气 
模型温度误差的分布特性.

文中采用TIMED/SABER 2.0版本 2 级温度数据作 为评估数据来源. 数据覆盖2002年1月-2016年12月共 计 15 年. 原始数据经过信息范围检查、极值检查、垂 直一致性检查等质量控制和统计学检查之后 ${ }^{[21,27]}$, 用 于对模式的统计评估. 在一个网格内, 经统计学检查 后剔除的数据比重近似满足莱特准则, 数据准确可靠. 在运行模式过程中，保持USSA-76和NRLMSISE-00模 式的输入与卫星观测点的经纬度、高度、时间一致, 获得所需对应位置相同条件下的模式数据. 根据 NRLMSISE-00模式的使用说明, 高度在 $80 \mathrm{~km}$ 以上, 太 阳活动和地磁活动对NRLMSISE-00模式计算的结果 影响较大, 故NRLMSISE-00模式的输入采用当日实际 $\mathrm{F} 107$ 和 $\mathrm{AP}$ 指数. 对于 $80 \mathrm{~km}$ 以下, 太阳活动和地磁活动 对NRLMSISE-00模式的计算结果无影响, F107, F107A和AP指数分别采用模式默认值进行计算.

\section{2 评估和分析方法}

为了评估模式与观测数据之间，不同高度和不同 纬度条件下的适用性, 分析比较主要采用以下几种 方式.

（1）日平均个例比较：以2016年4月 15 日卫星观测 数据为例, 对该日按高度分别求出各高度的平均温度 和标准偏差, 比较USSA-76和NRLMSISE-00模式日平 均温度与卫星观测日平均温度数据, 计算模式温度相 对于观测温度在 20-100 km高度的精度、温度偏差和 温度偏差的标准差.

平均温度定义为

$T(h)=\frac{1}{n} \sum_{i=1}^{n} T_{i}(h)$,

其中, $i$ 表示该日第 $i$ 条廓线, $T_{i}(h)$ 表示高度为 $h$ 的第 $i$ 条 廓线的温度值.

标准差定义为

$\sigma(h)=\sqrt{\frac{1}{n-1}\left\{\sum_{i=1}^{n}\left[T_{i}(h)-T(h)\right]^{2}\right\}}$.

温度偏差定义为

$\Delta T_{i}(h)=T_{i}(h)-T_{m}(h)$,

其中, $T_{m}$ 为对应条件下模式值.

温度偏差的标准差定义为
$\sigma_{\Delta T}(h)=\sqrt{\frac{1}{n-1}\left\{\sum_{i=1}^{n}\left[\Delta T_{i}(h)-\overline{\Delta T}(h)\right]^{2}\right\}}$.

模式精度定义为

$P(h)=\left(1-\frac{\left|T(h)-T_{m}(h)\right|}{\bar{T}(h)}\right) \times 100 \%$,

其中, $\bar{T}_{m}(h)$ 为在高度 $h$ 的模式平均值.

(2) 月平均个例比较: 以2016年1月卫星观测数据 为例, 类比日平均个例分析方法, 对该月按高度分别求 出各高度的平均温度和标准偏差, 比较USSA-76和 NRLMSISE-00模式月平均温度与卫星观测月平均温 度数据, 计算模式温度相对于观测温度在 20-100 km高 度的温度偏差、温度偏差的标准差和模式精度.

(3) 统计比较: 以 $\pm 30^{\circ} \mathrm{N}$ 和 $\pm 60^{\circ} \mathrm{N}$ 为分界线, 将纬度 划分为低纬、中纬和高纬地区. 参考万田等人 ${ }^{[12]}$ 计算 特定误差带下置信度的方法，基于 15 年的观测数据, 统计分析模式的置信度. 通过设置温度偏差, 统计温 度偏差要求下的样本数, 计算置信度, 评估不同纬度 地区两个大气模型的适用性, 分析温度偏差、温度偏 差的标准差和均方根误差的分布特征, 评估模式的适 用能力. 假设给定温度偏差 $\Delta T$, 总样本数 $N_{T}$, 统计获 得满足温度偏差要求的样本数为 $N_{X}$, 所求置信度为

$\alpha=\frac{N_{X}}{N_{T}} \times 100 \%$.

均方根误差定义为

$\operatorname{RMSE}(h)=\sqrt{\frac{1}{n} \sum_{i=1}^{n}\left(\Delta T_{i}(h)\right)^{2}}$.

\section{3 结果与讨论}

\section{1 日平均个例分析}

为分析USSA-76和NRLMSISE-00模式在以天为 单位的时间尺度的适用性, 统计分析了2016年4月15日 的温度廓线, 共计 1318 条. 对该日按高度分别求得各高 度上的温度标准偏差. NRLMSISE-00和USSA-76大气 模型温度的垂直廓线基本具有相同的变化特征, 在3040和50-70 km高度范围, 大气模型和卫星观测的温度 数据吻合较好. 同时, 利用大气模型预测的大气温度 数据和卫星观测的温度数据也存在一定的差异, 在 
40-50和70-100 km，两个大气模型温度廓线与卫星观 测的温度廓线差异较大，如图1(a)所示. 图1(b)给出了 两个大气模型相对于TIMED/SABER观测的平均温度 偏差随高度的变化曲线, NRLMSISE- 00 模式与 TIMED/SABER温度在 20-100 km范围内的平均偏差 基本小于 $10 \mathrm{~K}$, 在 $80 \mathrm{~km}$ 左右的局部区域, 平均温度偏 差超过 $10 \mathrm{~K}$. 在 20-30和 87-100 k m 高度范围, NRLMSISE-00呈现正偏差，最大正偏差为 $5.0 \mathrm{~K}$ ，表明 NRLMSISE-00模式预测的温度在该高度范围小于卫 星的观测温度; 在30-87 km高度范围, 模式温度与卫星 观测温度呈现负偏差, 最大负平均偏差为 $11.0 \mathrm{~K}$, 该范 围的模式温度大于卫星的观测温度. USSA-76模式与 TIMED/SABER温度平均偏差在较低高度偏差较小, 较高高度偏差较大. 在 $80 \mathrm{~km}$ 以下，模式温度与卫星观 测温度呈现负偏差, 最大负偏差为 $11.6 \mathrm{~K}$, 该范围的模 式温度大于卫星的观测温度; 在81-100 km，模式温度 与卫星观测温度呈现正偏差, 最大正偏差为 $25.9 \mathrm{~K}$.

模式温度与卫星观测温度偏差的标准差随着高度 的升高有明显的上升趋势, 在 $86 \mathrm{~km}$ 以下, NRLMSISE-
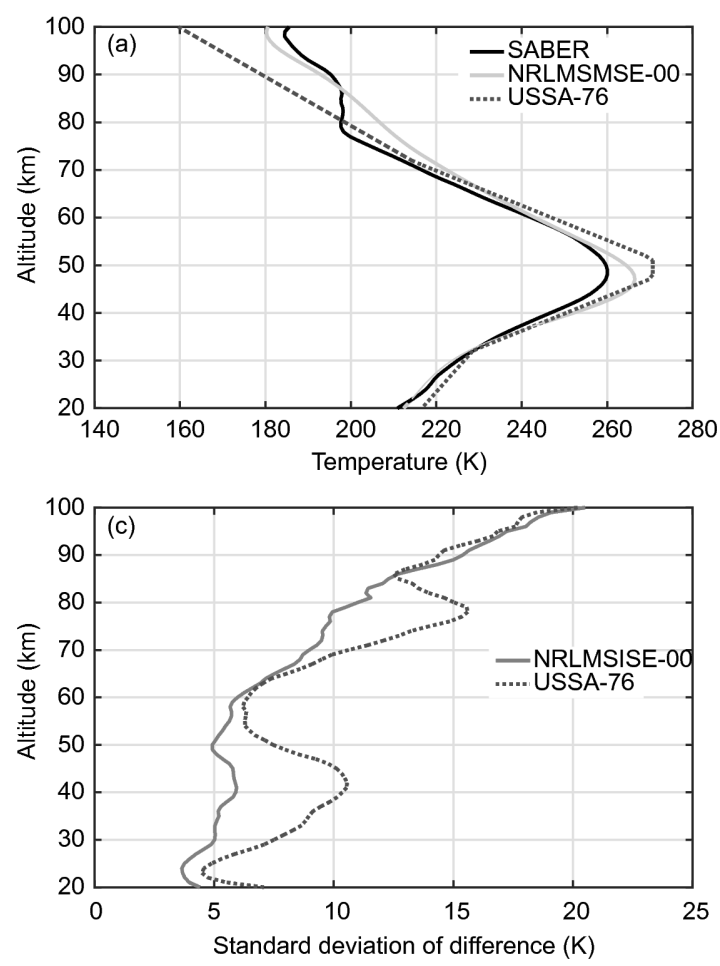

00 模式的标准差小于USSA-76的标准差, 在 $86 \mathrm{~km}$ 以 上，NRLMSISE-00模式的标准差大于USSA-76的标准 差, 如图1(c)所示. 图1(d)给出的是以 TIMED/SABER卫 星观测数据为标准, 分别计算出的两个大气模型的精 度, 用以定量评估两模式的优劣. $80 \mathrm{~km}$ 以上, NRLMSISE-00模式的精度明显优于USSA-76模式; $70 \mathrm{~km}$ 以下，NRLMSISE-00模式的精度在 $95 \%$ 以上， 20-100 km高度范围的平均精度为 $98.3 \%$. USSA-76模 式在 $80 \mathrm{~km}$ 以下，精度在 $95 \%$ 以上; 在 $80 \mathrm{~km}$ 以上, USSA-76模式的精度随高度降低, 在100 km高度降低 至 $86 \%$. 在 20-100 km 高度范围, USSA-76和 NRLMSISE-00模式的平均精度分别为 $96.7 \%$ 和 $98.3 \%$, 在以天为时间尺度的温度预测，USSA-76模式的精度 低于NRLMSISE-00模式的精度.

\section{2 月平均个例分析}

为分析USSA-76和NRLMSISE-00模式在以月为 单位的时间尺度的适用性, 统计分析了2016年1月的温 度廓线, 共计 43585 条. 两个大气模式的温度与卫星观
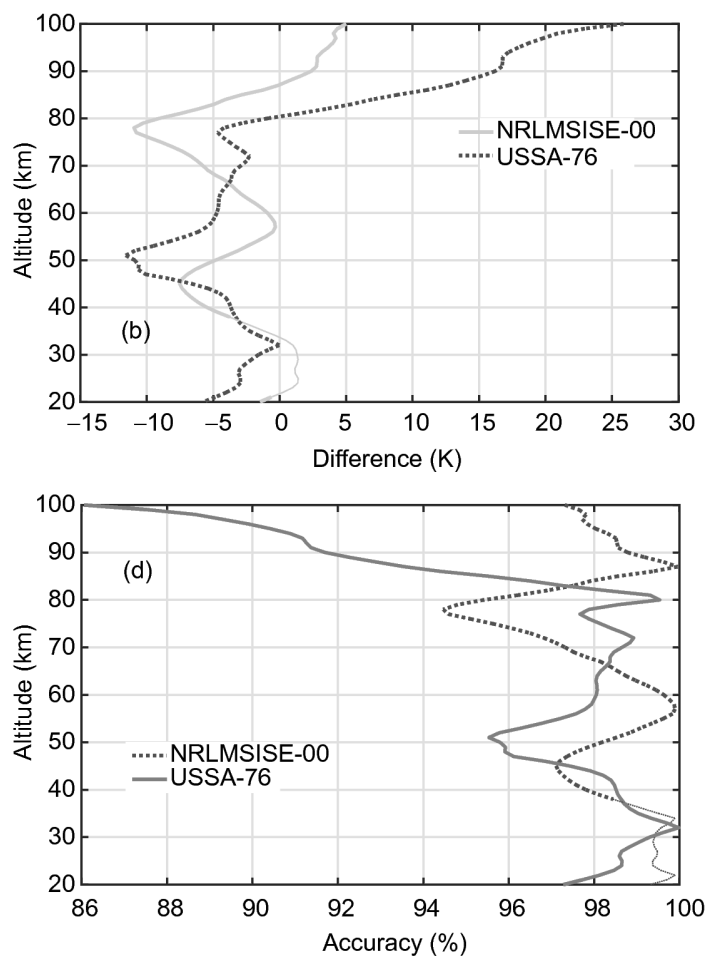

图 1 大气模式与卫星观测日平均温度个例比较结果. (a) 平均温度统计结果; (b) 平均温度偏差统计结果; (c) 温度偏差的标准 差统计结果; (d) 精度计算结果

Figure 1 A comparison between atmospheric models and SABER on daily mean temperature. (a) The mean temperature results; (b) the mean temperature differences; (c) the temperature standard deviation of differences; (d) the accuracy of atmospheric models. 
测的温度随高度的变化趋势也具有很好的一致性，如 图2(a)所示. NRLMSISE-00模式的平均温度偏差在 $87 \mathrm{~km}$ 以下基本呈现负偏差，表明该模式温度大于卫 星观测的温度, 最大负偏差为 $7.1 \mathrm{~K}$; 在 $24-31$ 和 $87 \mathrm{~km}$ 以上高度范围为正偏差，模式温度在该高度范围小于 卫星观测的温度，最大正偏差为6.3 K. USSA-76模式 的平均温度偏差在 29-42和83-94 km高度范围表现为 正偏差，最大正偏差为 $5.0 \mathrm{~K}$, 在其他高度范围呈现负 偏差, 最大负偏差为 $11.7 \mathrm{~K}$, 如图2(b)所示. 图2(c)是各 高度上温度偏差的标准差统计结果, NRLMSISE-00和 USSA-76模式具有相同的变化规律，温度偏差的标准 差随高度的变化廓线吻合较好. 在20-70 km高度范围, 温度偏差的标准差随高度变化较缓慢. $70 \mathrm{~km}$ 以上标准 差随高度变化较快，这与肖存英等人 ${ }^{[21]}$ 获得的温度标 准差随高度的变化一致，可能是由于不同高度的大气

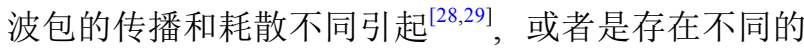
波模引起 ${ }^{[30]}$. 图2(d)给出的是两个模式的精度评估结 果，两个模式精度都在 $95 \%$ 以上，NRLMSISE-00模式 在 $75 \mathrm{~km}$ 处的精度最低，为 $96.5 \%$, USSA-76模式在
$52 \mathrm{~km}$ 处的精度最低，为 $95.5 \%$. 在 $20-100 \mathrm{~km}$ 高度范 围, USSA-76和NRLMSISE-00模式的平均精度分别为 $98.1 \%$ 和 $98.2 \%$, 在以月为时间尺度的温度预测, USSA76的精度略微低于NRLMSISE-00模式的精度.

\section{3 基于 15 年数据的统计比较}

(1) 平均温度偏差

为分析不同纬度地区大气模型与卫星观测的温度 偏差随高度和月份的变化，统计分析了 15 年各高度月 平均温度偏差, 结果如图3所示. 北半球高纬地区在 4 和8月及南半球高纬地区在 2,6 和10月, 卫星数据无法 覆盖该纬度区域, 无温度观测数据.

统计结果表明，大气模式与卫星观测的温度偏差 存在随高度和季节的变化特征. 北半球高纬地区 NRLMSISE-00模式在 20-70 km的温度偏差的绝对值 $\leq 15 \mathrm{~K}$, 在70 km以上, $5-7$ 月份的温度偏差在较高高度 约 $50 \mathrm{~K}$. 在中间层顶附近, NRLMSISE-00模式的温度 偏差在6和12月附近出现极值, 6月表现为正偏差, 12月 为负偏差. 同时, NRLMSISE- 00 模式温度偏差等值线
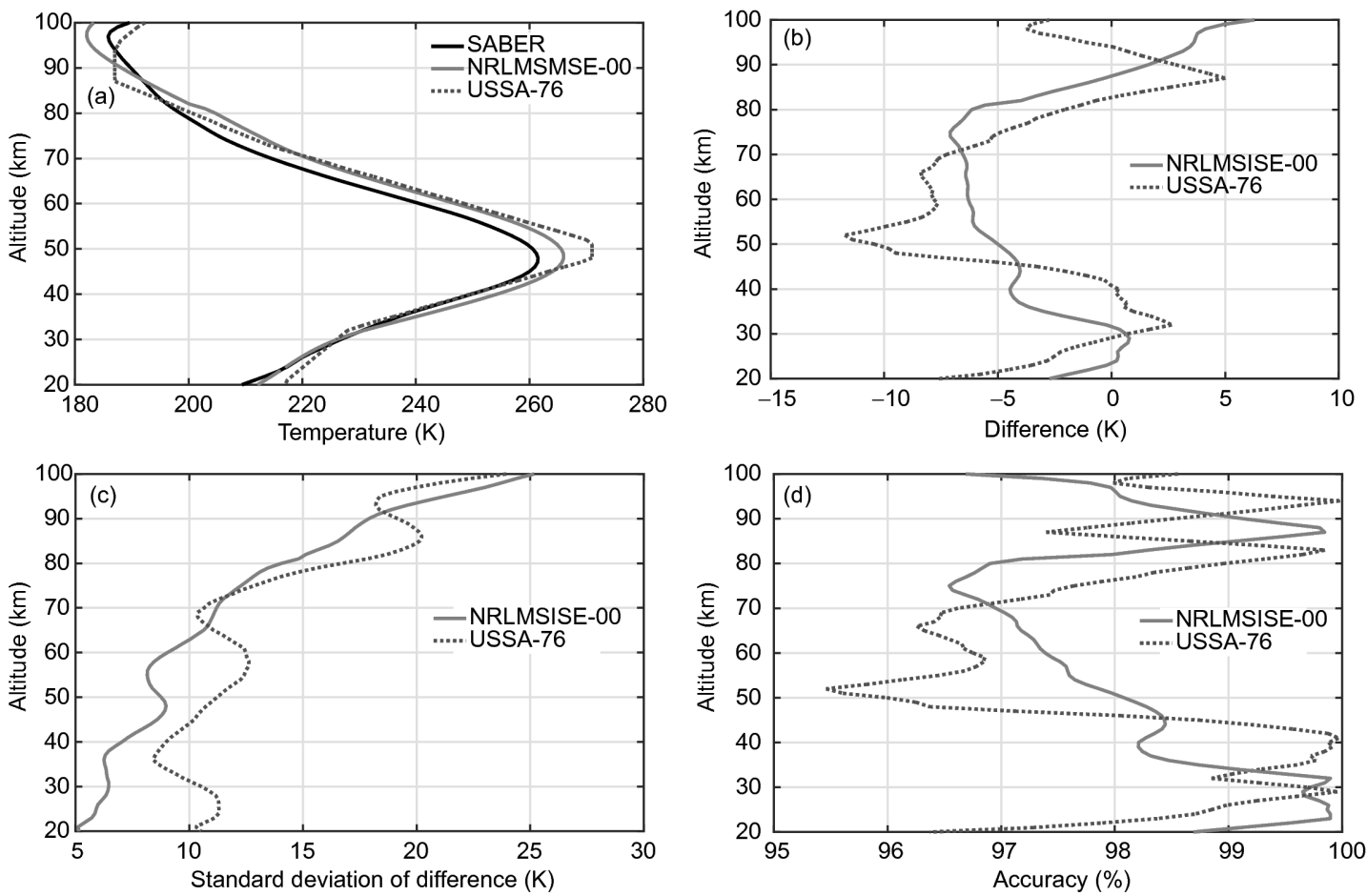

图 2 大气模式与卫星观测月平均温度个例比较结果. (a) 平均温度统计结果; (b) 平均温度偏差统计结果; (c) 温度偏差的标准 差统计结果; (d) 精度计算结果

Figure 2 A comparison between atmospheric models and SABER on monthly mean temperature. (a) The mean temperature results; (b) the mean temperature differences; (c) the temperature standard deviation of differences; (d) the accuracy of atmospheric models. 
(a) $@>60^{\circ} \mathrm{N}$

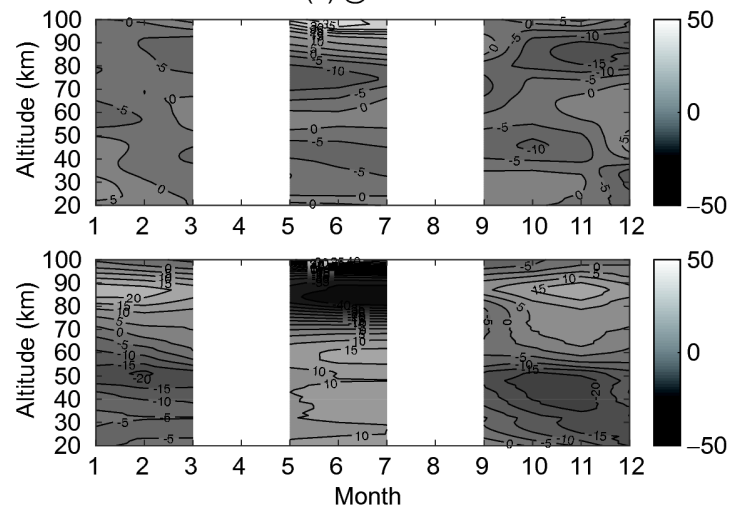

(c) @ $<(-60)^{\circ} \mathrm{N}$
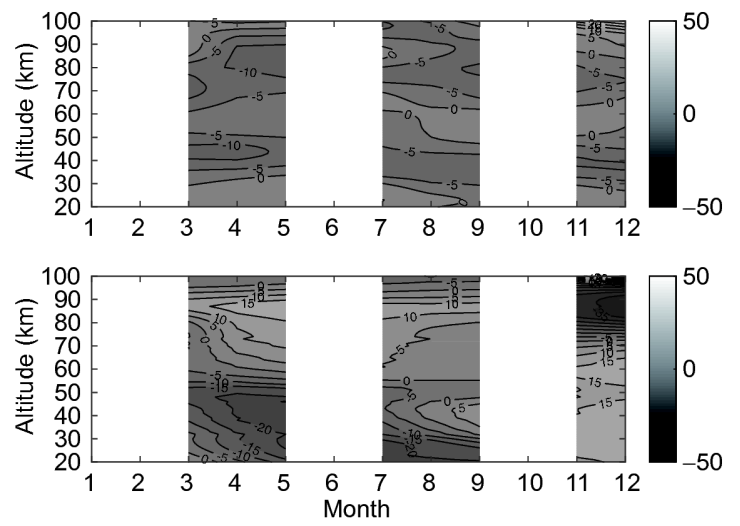

(b) $@ 30^{\circ} \mathrm{N}-60^{\circ} \mathrm{N}$

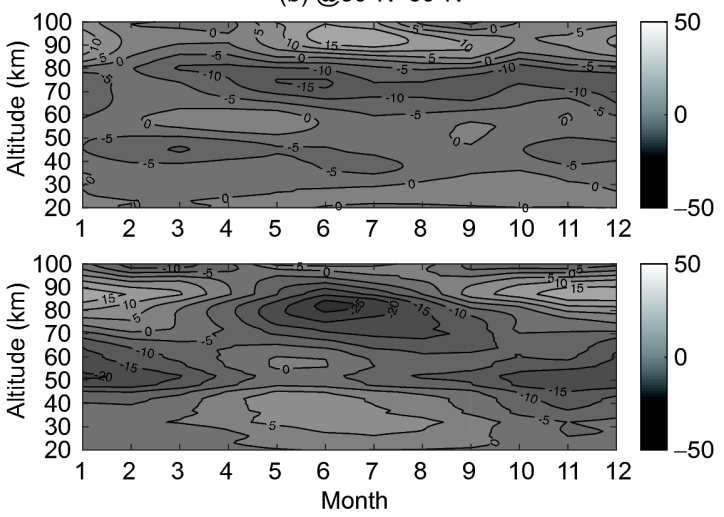

(d) @(-60 $\mathrm{N})-(-30)^{\circ} \mathrm{N}$

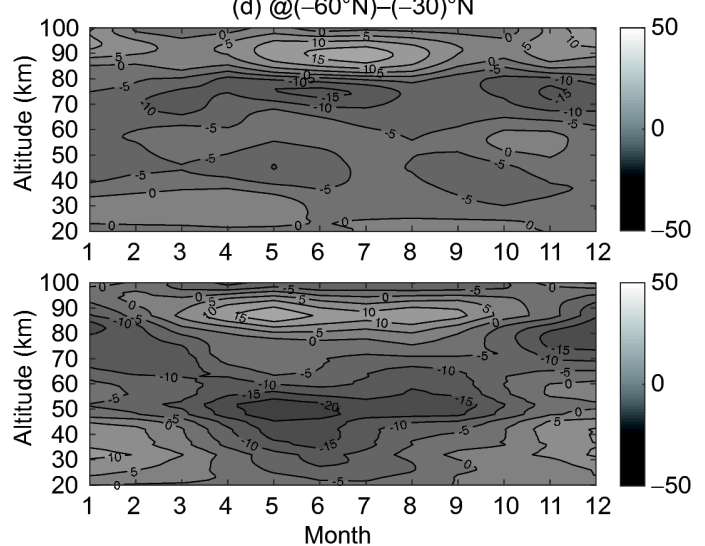

(e) @- $-30^{\circ} \mathrm{N}-30^{\circ} \mathrm{N}$
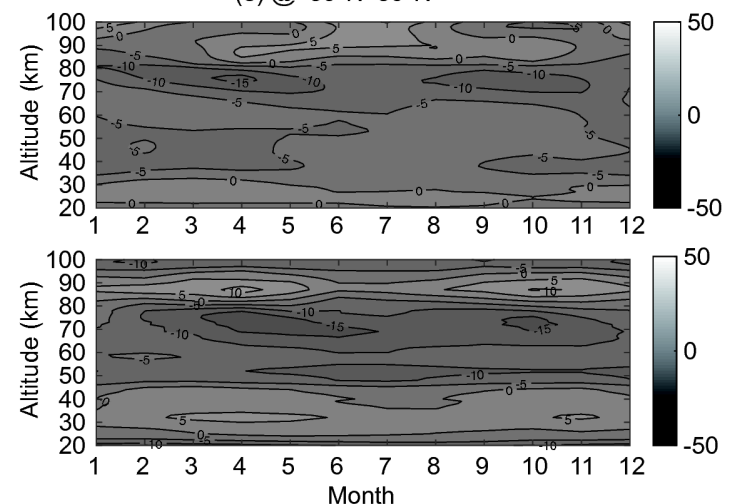

图 3 NRLMSISE-00(上)和USSA-76(下)与TIMED/SABER观测温度偏差在不同纬度地区随月份和高度的变化. (a) 北半球高 纬地区; (b) 北半球中纬地区; (c) 南半球高纬地区; (d) 南半球中纬地区; (e) 低纬地区

Figure 3 The temperature differences between the atmospheric models and SABER at high latitudes in (a) northern hemisphere and (c) southern hemisphere, middle latitudes in (b) northern hemisphere and (d) southern hemisphere and (e) low latitudes. The upper is NRLMSISE-00, lower is USSA-76.

在6和12月的 $70 \mathrm{~km}$ 以上较为密集, 表明温度偏差在该 高度范围内快速变化. USSA-76模式在20-70 km的温 度偏差的绝对值 $\leq 25 \mathrm{~K}$, 在平流层顶附近, USSA-76模 式温度偏差在6月份存在温度偏差极大值. 在 $70 \mathrm{~km}$ 以 上，温度偏差较大，在6月份中间层顶附近，温度偏差
存在极小值, 约为 $-50 \mathrm{~K}$. 在 2 和 11 月出现温度偏差极大 值, 约为 $15 \mathrm{~K}$.

在北半球中纬地区, NRLMSISE-00模式的温度偏 差在 $85 \mathrm{~km}$ 以下基本为负偏差, 在 $85 \mathrm{~km}$ 以上为正偏差. NRLMSISE-00模式温度偏差的绝对值 $\leq 20 \mathrm{~K}$. 在 6 月附 
近, 温度偏差在 75 和 $90 \mathrm{~km}$ 左右分别存在极小值和极大 值. USSA-76模式在 $40 \mathrm{~km}$ 以下, 温度偏差较小, 等值线 稀疏. 在 $70 \mathrm{~km}$ 以上温度偏差较大, 等值线密集. 在中间 层顶附近，温度偏差在6-7月存在极小值，约为 $-25 \mathrm{~K}$. 在12-1月温度偏差存在极大值, 约为 $15 \mathrm{~K}$.

在低纬地区，NRLMSISE-00模式的温度偏差基本 呈现负偏差，温度偏差的绝对值 $\leq 20 \mathrm{~K}$. USSA-76模式 的温度偏差在 $45 \mathrm{~km}$ 以下以正偏差为主，在 4 和 11 月的 $30 \mathrm{~km}$ 处存在温度偏差极大值, 约为 5 K. 45- $80 \mathrm{~km}$, 温 度偏差呈现负偏差, 在 4,5 和 10 月存在温度偏差的极小 值, 约为-15 K. 80-100 km, 温度偏差等值线密集分布, 尤其在 4 和 10 月的 $85 \mathrm{~km}$ 高度, 温度偏差存在极大值, 约 为 $10 \mathrm{~K}$.

在南半球中纬地区, NRLMSISE-00模式温度偏差 随月份和高度的分布与北半球中纬度地区的分布类 似. NRLMSISE-00模式的温度偏差在 5-7月的中间层 顶附近变化较大. 中纬度地区USSA-76模式的温度偏 差在南北半球的分布不同. 在中间层顶和平流层顶附 近，温度偏差分别以正偏差和负偏差为主，都在 5 月份 附近存在极值. 对比两半球中纬度地区USSA-76模式 的温度偏差分布，在中间层顶和平流层顶附近，4-9月 两半球温度偏差符号相反.

在南半球高纬地区, NRLMSISE-00模式的温度偏 差整体较小. USSA-76模式的温度偏差在UMLT附近 较大, 尤其在11-12月份温度偏差约-50 K, 说明USSA76 模型在该季节和高度的适用性较差. 从USSA-76模 式建模方法角度来说，代表的是北半球中纬度地区理 想大气的平均状态，由一条廓线来表示全球大气状态， 故该模式在表征不同季节的温度变化上具有很大的局 限性, 模式值与观测值在南半球高纬地区差异明显.

NRLMSISE-00模式在UMLT高度的温度偏差比低 高度的温度偏差大, 尤其是较高纬度地区. 建模的数据 来源在较高纬度地区主要来自于落球数据、压力表数 据等, 数据的缺乏 ${ }^{[6,10]}$ 可能是导致温度偏差较大的原因 之一.

大气波动是大气温度的重要扰动源, 如重力波、 行星波和潮汐波等. 在中高纬度中间层高度，行星波 的振幅可以达到10-15 $\mathrm{K}^{[21,31]}$. 在中间层上部，重力波 的振幅也可达到10-15 K 左右 ${ }^{[32]}$. 在中间层上部和低热 层(UMLT)高度，大气潮汐对温度的变化起着主导作 用，尤其是赤道附近，潮汐波的振幅达到10-15 K. 中
纬度地区20-45 km，波数为1-3的行星波起重要作用， 温度的扰动与浴流热通量高度相关 ${ }^{[28]}$. 图 3 可以发现, 模式与卫星观测的温度偏差较大的区域，对应着大气 波动较强的区域, 说明模式对波动的表征有待进一步 改进, 需要对不同区域波动的参数化方案进行优化.

(2) 温度偏差的标准差

大气模式的平均温度偏差能反映大气模式与真实 大气整体的误差的离散程度, 为分析大气模式与卫星 观测温度偏差在不同高度和月份的离散程度，统计分 析了不同纬度地区温度偏差的标准差随月份和高度的 变化, 如图4所示. NRLMSISE-00模式温度偏差的标准 差与USSA-76模式温度偏差的标准差随月份和高度的 变化具有相似的结构. 在低热层, 同一模式温度偏差的 标准差等值线随高度逐渐变密, 说明随着高度的升高, 温度偏差的标准差的增长速率越快, 在低热层以下, 温 度偏差的标准差等值线稀疏，温度偏差的标准差随高 度的变化缓慢. 与图2(c)结果一致, 温度偏差的标准差 在 $70 \mathrm{~km}$ 以下呈线性增长, 在 $70 \mathrm{~km}$ 以上近似呈指数增 长. 同一模式, 高度越高, 温度偏差的标准差越大, 温 度偏差随高度分布越离散. 在 $80 \mathrm{~km}$ 以下, 两个半球的 中纬度地区温度偏差的标准差随月份和高度的分布有 所不同. 在北半球中纬度地区, 相同高度下7月份附近 的温度偏差的标准差略小于12月附近的温度偏差标准 差. 在南半球中纬度地区, 相同高度下, 7 月份附近的温 度偏差的标准差略大于 12 月附近的温度偏差的标准 差. 两个半球冬季的温度偏差的标准差大于夏季的温 度偏差的标准差.

(3) 均方根误差

为更直观地反映不同大气模式表征真实大气的能 力，统计了大气模式与卫星观测温度之间的均方根误 差，如图5所示. 均方根误差越大，大气模型在该区域 的表征能力越差, 反之, 表征能力越强. 在北半球高纬 度地区, 相同月份和高度下, USSA-76模式的均方根误 差比NRLMSISE-00模式的均方根误差大. 尤其在中间 层顶和平流层顶附近，NRLMSISE-00模式明显优于 USSA-76模式，NRLMSISE-00模式相比USSA-76模式 更接近实际大气.

在北半球中纬度地区，NRLMSISE-00模式在夏季 UMLT高度的均方根误差较大，模式表征真实大气的 能力较差. 在平流层和中间层, 相同高度下, 冬季的均 方根误差比其他季节的均方根误差大. USSA-76模式 
(a) $@>60^{\circ} \mathrm{N}$
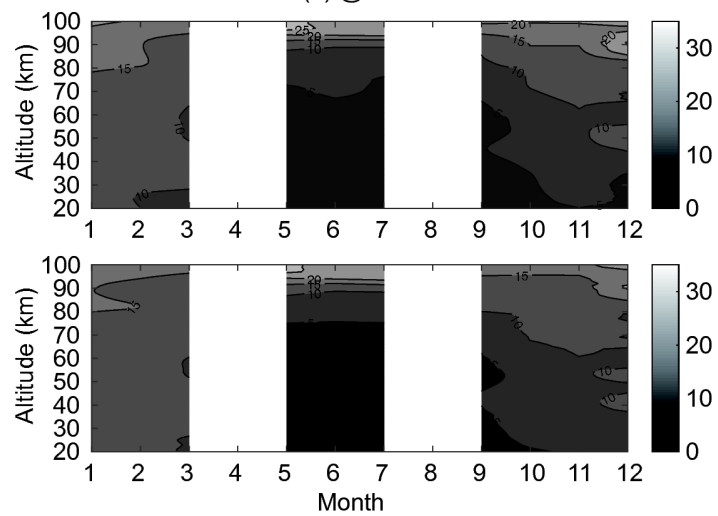

(c) @ $<(-60)^{\circ} \mathrm{N}$
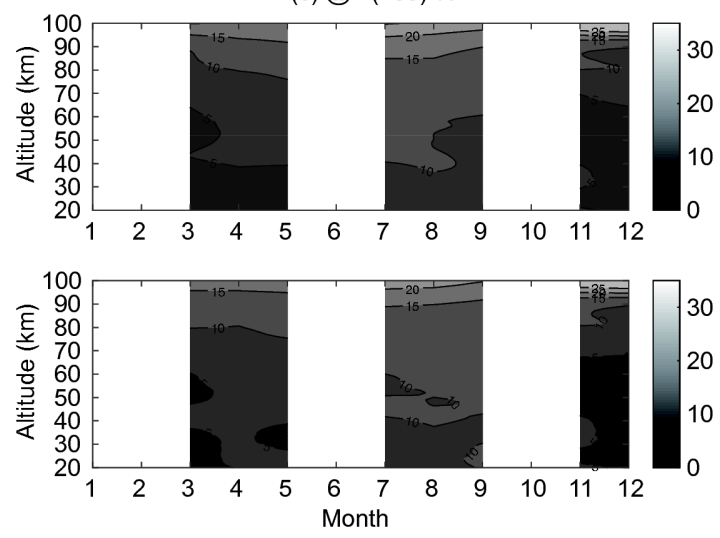

(b) @30 $\mathrm{N}-60^{\circ} \mathrm{N}$
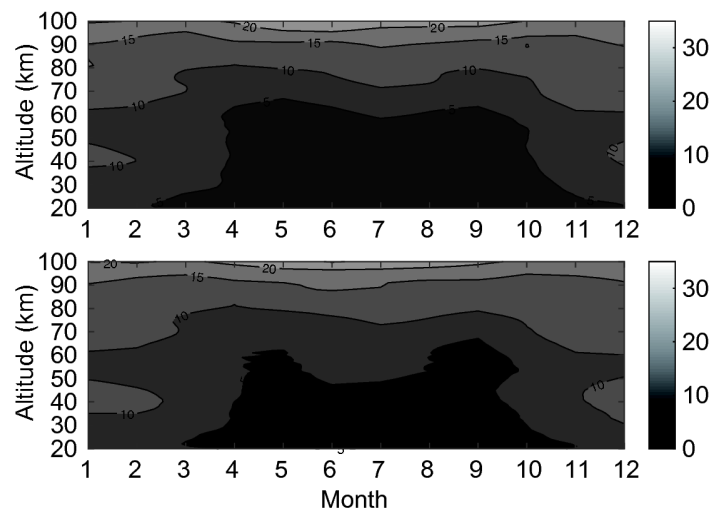

(d) @(-60' $\mathrm{N})-(-30)^{\circ} \mathrm{N}$
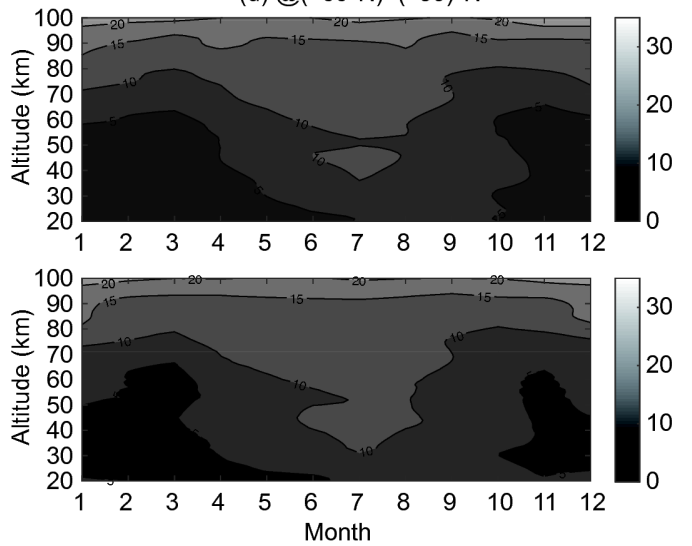

(e) $@-30^{\circ} \mathrm{N}-30^{\circ} \mathrm{N}$
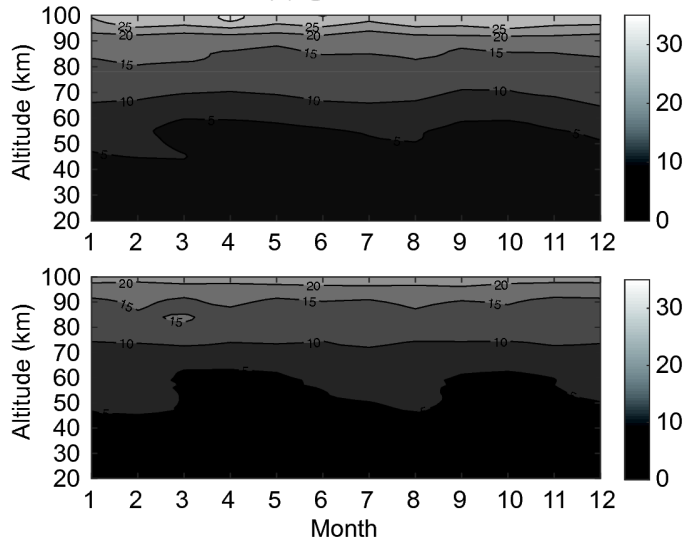

图 4 NRLMSISE-00(上)和USSA-76(下)与TIMED/SABER温度偏差的标准差在不同纬度地区随月份和高度的变化. (a) 北半 球高纬地区; (b) 北半球中纬地区; (c) 南半球高纬地区; (d) 南半球中纬地区; (e) 低纬地区

Figure 4 The temperature standard deviations of differences between the atmospheric models and SABER at high latitudes in (a) northern hemisphere and (c) southern hemisphere, middle latitudes in (b) northern hemisphere and (d) southern hemisphere and (e) low latitudes. The upper is NRLMSISE-00, lower is USSA-76.

在UMLT高度冬夏季和中间层冬季均方根误差较大, 尤其在平流层顶和中间层顶附近. USSA-76模式不能 很好地处理在平流层顶和中间层顶温度过渡区的温度 表征能力.
在南半球高纬度地区, 两个大气模式在夏季 UMLT高度均方根误差较大. 在南半球的中纬度地区, 中间层冬季的均方根误差大于相同高度下其他月份的 均方根误差. 
(a) @ $>60^{\circ} \mathrm{N}$

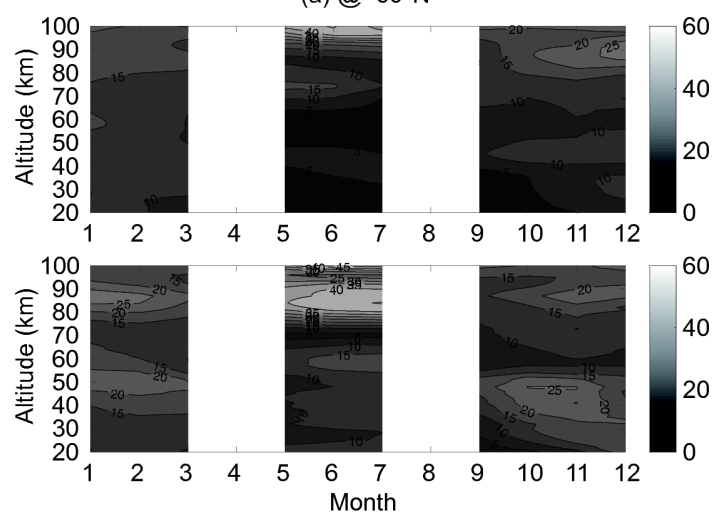

(c) @ $<(-60)^{\circ} \mathrm{N}$

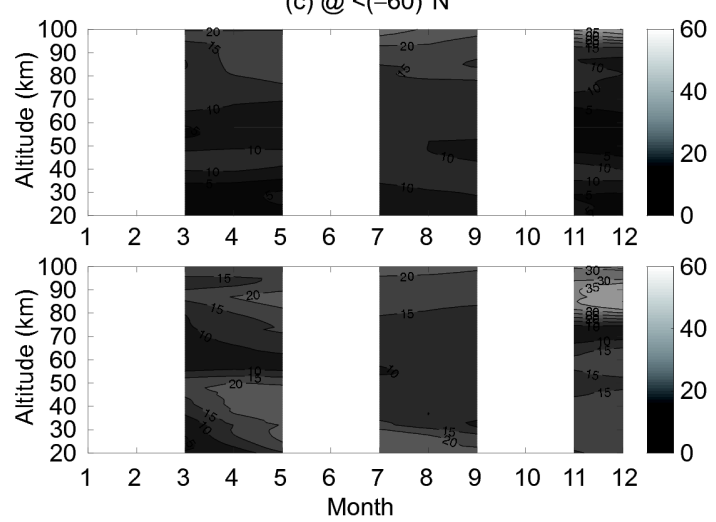

(b) $@ 30^{\circ} \mathrm{N}-60^{\circ} \mathrm{N}$
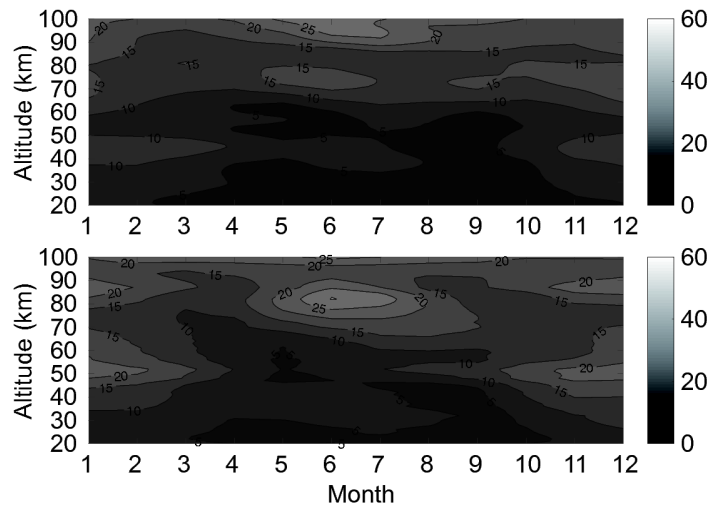

(d) @(-60 $\mathrm{N})-(-30)^{\circ} \mathrm{N}$
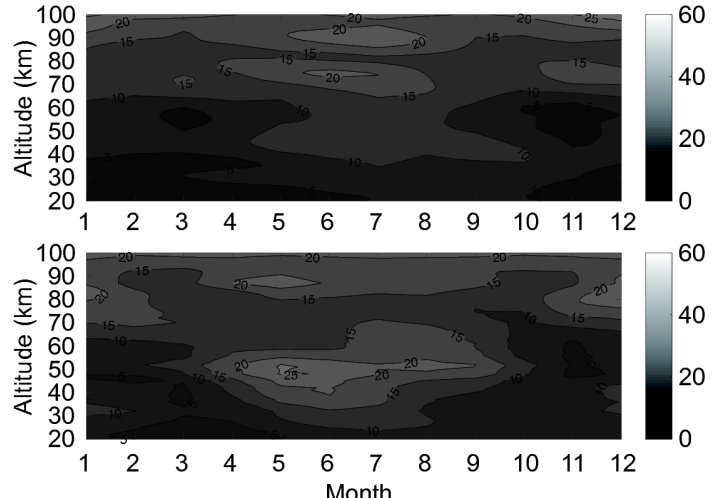

(e) @- $30^{\circ} \mathrm{N}-30^{\circ} \mathrm{N}$

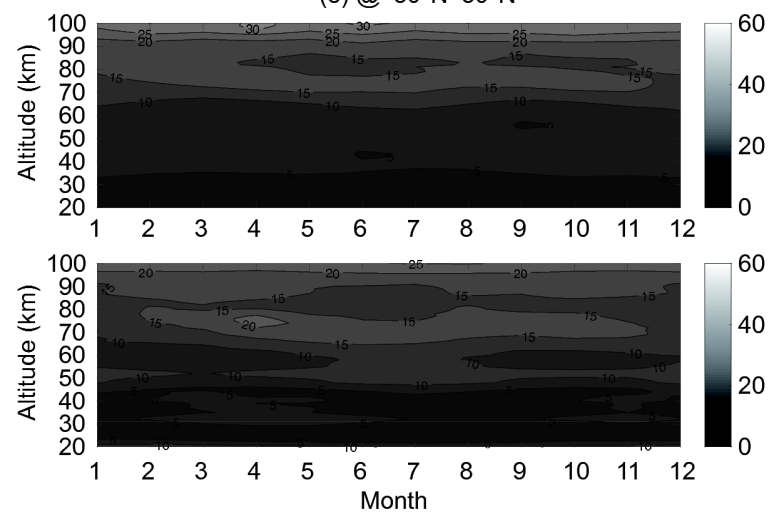

图 5 NRLMSISE-00(上)和USSA-76(下)与TIMED/SABER观测温度的均方根误差在不同纬度地区随月份和高度的变化. (a) 北半球高纬地区; (b) 北半球中纬地区; (c) 南半球高纬地区; (d) 南半球中纬地区; (e) 低纬地区

Figure 5 The temperature root mean square error between the atmospheric models and SABER at high latitudes in (a) northern hemisphere and (c) southern hemisphere, middle latitudes in (b) northern hemisphere and (d) southern hemisphere and (e) low latitudes. The upper is NRLMSISE-00, lower is USSA-76.

在低纬度地区，平流层和中间层下部两个大气模 式的均方根误差在 15 K以下. NRLMSISE-00模式在低 热层的均方根误差最大, 达到 $25 \mathrm{~K}$ 以上. USSA-76模式 在UMLT高度, 均方根误差较大. 两个大气模式的均方 根误差在低纬地区无明显的季节变化.
(4) 置信度

(a) 不同纬度地区的变化

为了分析USSA-76和NRLMSISE-00模式在不同 纬度区域的适用性, 划分了 5 个纬度带, 如表1所示. 通 过设置温度偏差, 统计温度偏差要求下的样本数, 计算 
不同纬度地区不同温度偏差要求下两个大气模式的置 信度，以便分别对不同纬度地区大气模式的适用性进 行分析, 结果如表2所示. USSA-76模式和NRLMSISE00 模式的置信度在不同纬度地区具有相同的规律．在 相同温度偏差要求下，低纬地区的置信度最高，其次 是中纬地区的置信度, 高纬地区的置信度最低. 其中, USSA-76模式的数据来自于中低纬探空火箭的探测数 据, 不足以反映高纬地区的大气状态, USSA-76模式在 两半球高纬地区的置信度较低. 当温度偏差 $\leq 20 \mathrm{~K}$ 时, 相同温度偏差下，北半球中纬度地区的置信度高于南 半球中纬度地区的置信度. 当温度偏差 $>20 \mathrm{~K}$ 时，相同 温度偏差下，在中纬度地区大气模式在两个半球的置 信度相当. 对比两个大气模式，在相同的温度偏差和 相同纬度地区下，NRLMSISE-00模式的置信度高于相 同条件下USSA-76模式的置信度.

NRLMSISE-00模式的数据来源比USSA-76模式 多, 覆盖范围相比USSA-76的数据覆盖范围大, 利用低 阶球谐函数的展开表示大气温度的时空变化，模型中 也加入了太阳活动和地磁活动等影响，相比USSA-76 模式的平均气候状态, NRLMSISE-00能够更好地反映 真实的大气状态, 故NRLMSISE-00模式的置信度在通 常情况下高于同等条件下USSA-76模式的置信度.

(b) 不同高度的变化

表3统计分析了USSA-76模式在不同纬度地区典 型高度的置信度, 以 $30 \mathrm{~km}$ 高度代表平流层, $65 \mathrm{~km}$ 代表 中间层, $90 \mathrm{~km}$ 代表低热层. 在高纬度地区, 当温度偏差
表 1 纬度划分情况

Table 1 Latitude division

\begin{tabular}{cc}
\hline 纬度划分 & 纬度范围(N) \\
\hline 低纬地区 & $-30^{\circ}-30^{\circ}$ \\
中纬地区 & $-60^{\circ}--30^{\circ} ; 30^{\circ}-60^{\circ}$ \\
高纬地区 & $<-60^{\circ} ;>60^{\circ}$ \\
\hline
\end{tabular}

$\leq 20 \mathrm{~K}$ 时，相同温度偏差下，USSA-76模式在 $65 \mathrm{~km}$ 处 的置信度最高. 在南半球, $90 \mathrm{~km}$ 的置信度大于 $30 \mathrm{~km}$ 的置信度. 在北半球, $90 \mathrm{~km}$ 的置信度小于 $30 \mathrm{~km}$ 的置 信度. 在中纬度地区, 相同温度偏差下, USSA-76模式 的置信度随着高度的升高而降低. 在低纬地区, 当温度 偏差 $\geq 15 \mathrm{~K}$ 时, $30 \mathrm{~km}$ 的置信度最大, $90 \mathrm{~km}$ 的置信度最 小; 当温度偏差 $<15 \mathrm{~K}$ 时, $65 \mathrm{~km}$ 的置信度最小. 相同温 度偏差下, 对比不同纬度地区 $30 \mathrm{~km}$ 的置信度, 结果表 明, 低纬地区置信度最高, 其次中纬地区, 高纬地区的 置信度最小. USSA-76模式在北半球中纬度地区 $30 \mathrm{~km}$ 的置信度高于相同条件下南半球的置信度，在高纬地 区 $30 \mathrm{~km}$ 高度也具有相同的规律. 在 $65 \mathrm{~km}$ 高度，当温 度偏差 $>20 \mathrm{~K}$ 时，相同温度偏差下，不同纬度地区下的 置信度差别不大; 当温度偏差 $\leq 20 \mathrm{~K}$ 时, 相同温度偏差 下, 低纬地区的置信度最低, 南半球中纬度地区置信度 最高, 北半球高纬地区的置信度次之. 在 $90 \mathrm{~km}$ 高度, 相 同温度偏差下, 低纬地区的置信度最高. 在相同温度偏 差和相同纬度区域下，南半球的置信度高于相同条件 下北半球的置信度.

表 2 满足不同温度偏差下的置信度

Table 2 Confidence level of atmospheric models in different temperature deviations

\begin{tabular}{|c|c|c|c|c|c|c|c|c|}
\hline \multirow{2}{*}{ 模式 } & \multirow{2}{*}{ 纬度划分 } & \multicolumn{7}{|c|}{ 温度偏差 $\Delta T(\mathrm{~K})$} \\
\hline & & \pm 5 & \pm 10 & \pm 15 & \pm 20 & \pm 30 & \pm 40 & \pm 50 \\
\hline \multirow{5}{*}{ USSA-76 } & 高纬(N) & $18.5 \%$ & $40.0 \%$ & $62.8 \%$ & $76.5 \%$ & $90.9 \%$ & $96.5 \%$ & $99.0 \%$ \\
\hline & 中纬(N) & $32.9 \%$ & $58.8 \%$ & $74.8 \%$ & $85.4 \%$ & $95.8 \%$ & $99.0 \%$ & $99.8 \%$ \\
\hline & 低纬 & $38.4 \%$ & $65.0 \%$ & $81.2 \%$ & $90.1 \%$ & $97.2 \%$ & $99.2 \%$ & $99.8 \%$ \\
\hline & 中纬(S) & $31.0 \%$ & $56.6 \%$ & $74.2 \%$ & $85.4 \%$ & $96.3 \%$ & $99.3 \%$ & $99.8 \%$ \\
\hline & 高纬(S) & $19.2 \%$ & $37.7 \%$ & $56.6 \%$ & $76.3 \%$ & $93.6 \%$ & $97.4 \%$ & $99.3 \%$ \\
\hline \multirow{5}{*}{ NRLMSISE-00 } & 高纬(N) & $40.3 \%$ & $65.5 \%$ & $80.4 \%$ & $89.2 \%$ & $96.5 \%$ & $98.6 \%$ & $99.4 \%$ \\
\hline & 中纬(N) & $44.8 \%$ & $68.9 \%$ & $82.2 \%$ & $90.2 \%$ & $97.2 \%$ & $99.3 \%$ & $99.8 \%$ \\
\hline & 低纬 & $45.7 \%$ & $70.3 \%$ & $82.4 \%$ & $89.2 \%$ & $96.0 \%$ & $98.5 \%$ & $99.4 \%$ \\
\hline & 中纬(S) & $40.5 \%$ & $66.4 \%$ & $81.1 \%$ & $89.8 \%$ & $97.2 \%$ & $99.2 \%$ & $99.8 \%$ \\
\hline & 高纬(S) & $39.8 \%$ & $66.6 \%$ & $82.8 \%$ & $91.1 \%$ & $97.2 \%$ & $98.9 \%$ & $99.5 \%$ \\
\hline
\end{tabular}


表 3 USSA-76模式在不同纬度地区和不同温度偏差下, 高度为 30,65 和 $90 \mathrm{~km}$ 的置信度

Table 3 The confidence level of USSA-76 at different altitudes $(30,65,90 \mathrm{~km})$, latitudes and temperature deviations

\begin{tabular}{|c|c|c|c|c|c|c|c|c|}
\hline \multirow{2}{*}{ 纬度 } & \multirow{2}{*}{ 高度(km) } & \multicolumn{7}{|c|}{ 温度偏差 $\Delta T(\mathrm{~K})$} \\
\hline & & \pm 5 & \pm 10 & \pm 15 & \pm 20 & \pm 30 & \pm 40 & \pm 50 \\
\hline \multirow{3}{*}{ 高纬(N) } & 30 & $16.9 \%$ & $48.5 \%$ & $76.7 \%$ & $88.6 \%$ & $100 \%$ & $100 \%$ & $100 \%$ \\
\hline & 65 & $26.2 \%$ & $56.1 \%$ & $82.3 \%$ & $93.4 \%$ & $99.2 \%$ & $100 \%$ & $100 \%$ \\
\hline & 90 & $13.2 \%$ & $27.2 \%$ & $41.3 \%$ & $54.6 \%$ & $75.1 \%$ & $87.9 \%$ & $96.1 \%$ \\
\hline \multirow{3}{*}{ 中纬(N) } & 30 & $50.1 \%$ & $87.6 \%$ & $95.1 \%$ & $98.3 \%$ & $100 \%$ & $100 \%$ & $100 \%$ \\
\hline & 65 & $25.8 \%$ & $51.6 \%$ & $75.0 \%$ & $90.0 \%$ & $98.0 \%$ & $100 \%$ & $100 \%$ \\
\hline & 90 & $22.6 \%$ & $43.5 \%$ & $61.2 \%$ & $74.9 \%$ & $91.3 \%$ & $97.6 \%$ & $99.6 \%$ \\
\hline \multirow{3}{*}{ 低纬 } & 30 & $72.2 \%$ & $99.1 \%$ & $100 \%$ & $100 \%$ & $100 \%$ & $100 \%$ & $100 \%$ \\
\hline & 65 & $17.5 \%$ & $44.4 \%$ & $73.2 \%$ & $91.3 \%$ & $99.6 \%$ & $100 \%$ & $100 \%$ \\
\hline & 90 & $26.3 \%$ & $49.5 \%$ & $67.7 \%$ & $80.6 \%$ & $94.2 \%$ & $98.7 \%$ & $99.8 \%$ \\
\hline \multirow{3}{*}{ 中纬(S) } & 30 & $40.4 \%$ & $73.5 \%$ & $89.9 \%$ & $96.2 \%$ & $100 \%$ & $100 \%$ & $100 \%$ \\
\hline & 65 & $27.7 \%$ & $55.6 \%$ & $78.3 \%$ & $90.6 \%$ & $98.4 \%$ & $100 \%$ & $100 \%$ \\
\hline & 90 & $24.1 \%$ & $45.9 \%$ & $64.0 \%$ & $77.6 \%$ & $92.9 \%$ & $98.2 \%$ & $99.7 \%$ \\
\hline \multirow{3}{*}{ 高纬(S) } & 30 & $8.8 \%$ & $19.8 \%$ & $37.7 \%$ & $70.9 \%$ & $100 \%$ & $100 \%$ & $100 \%$ \\
\hline & 65 & $25.3 \%$ & $47.6 \%$ & $71.6 \%$ & $92.9 \%$ & $99.9 \%$ & $100 \%$ & $100 \%$ \\
\hline & 90 & $15.8 \%$ & $31.4 \%$ & $46.0 \%$ & $58.4 \%$ & $75.8 \%$ & $87.5 \%$ & $96.3 \%$ \\
\hline
\end{tabular}

与表3类似，表4统计分析了NRLMSISE-00模式在 不同纬度地区典型高度的置信度。相同温度偏差下， NRLMSISE- 00 模式在 $30 \mathrm{~km}$ 的置信度最高，其次是 $65 \mathrm{~km}$ 的置信度，90 km的置信度最低，也就是在相同 纬度地区相同温度偏差下，置信度随着高度的升高而 降低. 在高纬地区，相同高度相同温度偏差下，南半球 的置信度高于北半球的置信度. 在中纬度地区, 相同高 度相同温度偏差下，北半球的置信度高于南半球的置 信度.

对比表 3 和 4 , 在北半球中纬度地区 $90 \mathrm{~km}$ 高度，当 温度偏差 $\leq 20 \mathrm{~K}$ 时, 相同温度偏差下, NRLMSISE-00模 式的置信度略低于USSA-76模式. 此外, 在低纬和南半 球中纬度地区的 $90 \mathrm{~km}$ 高度，NRLMSISE-00模式的置 信度低于相同条件下USSA-76模式的置信度。除上述 部分区域和高度外，NRLMSISE-00模式的置信度均高 于同等条件下USSA-76模式的置信度.

在低纬和南半球中纬地区 $90 \mathrm{~km}$ 高度统计出的 NRLMSISE-00模式置信度略低于USSA-76模式的置 信度. 上述结果与通常情况下的结果不同, 可能是因为 NRLMSISE-00模式从建模方法上来说表征的是大气 的气候状态，以典型高度 $90 \mathrm{~km}$ 为代表的低热层高度,
NRLMSISE- 00 模式反映的低纬度和南半球中纬度地 区大气气候态与真实大气瞬时状态有显著差异. 在后 续对低热层高度的模型修正或者建模中，需要对建模 方法作深入改进以提高NRLMSISE-00模式在低热层 大气的表征能力.

\section{4 结论}

本文基于 TIMED/SABER从2002年1月-2016年12 月的温度数据，对USSA-76和NRLMSISE-00模式在全 球临近空间20-100 km高度范围的精度和适用性进行 了评估, 得到如下结论.

(1) 两个大气模式与TIMED/SABER卫星观测的 温度随高度变化特性基本吻合，细节差异主要由模式 数据源、模型理论引起.

(2) USSA-76模式与卫星观测的日平均最大温度 偏差为 $25.9 \mathrm{~K}$, 模式的平均精度为 $96.7 \%$. 月平均最大 温度偏差为 $11.7 \mathrm{~K}$, 模式的月平均精度为 $98.1 \%$. NRLMSISE-00模式与卫星观测的日平均最大温度偏 差为 $110 \mathrm{~K}$, 模式的平均精度为 $98.3 \%$, 月平均最大平均 温度偏差为 $7.1 \mathrm{~K}$ ，平均精度为 $98.2 \%$; USSA-76和 NRLMSISE-00模式与TIMED/SABER温度偏差的标准 
表 4 NRLMSISE-00模式在不同纬度地区和不同温度偏差下, 高度为 30,65 和 $90 \mathrm{~km}$ 的置信度

Table 4 The confidence level of NRLMSISE-00 at different altitudes $(30,65,90 \mathrm{~km})$, latitudes and temperature deviations

\begin{tabular}{|c|c|c|c|c|c|c|c|c|}
\hline \multirow{2}{*}{ 纬度 } & \multirow{2}{*}{ 高度 $(\mathrm{km})$} & \multicolumn{7}{|c|}{ 温度偏差 $\Delta T(\mathrm{~K})$} \\
\hline & & \pm 5 & \pm 10 & \pm 15 & \pm 20 & \pm 30 & \pm 40 & \pm 50 \\
\hline \multirow{3}{*}{ 高纬(N) } & 30 & $66.5 \%$ & $83.6 \%$ & $91.8 \%$ & $96.3 \%$ & $99.2 \%$ & $99.9 \%$ & $100 \%$ \\
\hline & 65 & $40.8 \%$ & $71.9 \%$ & $87.6 \%$ & $95.0 \%$ & $99.4 \%$ & $100 \%$ & $100 \%$ \\
\hline & 90 & $24.2 \%$ & $45.6 \%$ & $63.1 \%$ & $76.5 \%$ & $92.2 \%$ & $98.0 \%$ & $99.6 \%$ \\
\hline \multirow{3}{*}{ 中纬(N) } & 30 & $75.4 \%$ & $92.9 \%$ & $98.2 \%$ & $99.6 \%$ & $99.9 \%$ & $100 \%$ & $100 \%$ \\
\hline & 65 & $34.5 \%$ & $64.9 \%$ & $84.7 \%$ & $93.9 \%$ & $99.4 \%$ & $100 \%$ & $100 \%$ \\
\hline & 90 & $21.6 \%$ & $41.8 \%$ & $59.4 \%$ & $73.6 \%$ & $91.3 \%$ & $98.0 \%$ & $99.7 \%$ \\
\hline \multirow{3}{*}{ 低纬 } & 30 & $90.2 \%$ & $99.8 \%$ & $100 \%$ & $100 \%$ & $100 \%$ & $100 \%$ & $100 \%$ \\
\hline & 65 & $37.7 \%$ & $67.7 \%$ & $86.3 \%$ & $95.4 \%$ & $99.8 \%$ & $100 \%$ & $100 \%$ \\
\hline & 90 & $22.6 \%$ & $43.3 \%$ & $60.8 \%$ & $74.4 \%$ & $91.0 \%$ & $97.5 \%$ & $99.5 \%$ \\
\hline \multirow{3}{*}{ 中纬(S) } & 30 & $60.9 \%$ & $91.4 \%$ & $97.7 \%$ & $99.5 \%$ & $99.9 \%$ & $100 \%$ & $100 \%$ \\
\hline & 65 & $30.6 \%$ & $61.7 \%$ & $83.1 \%$ & $93.0 \%$ & $99.0 \%$ & $100 \%$ & $100 \%$ \\
\hline & 90 & $21.5 \%$ & $41.6 \%$ & $59.2 \%$ & $73.5 \%$ & $91.1 \%$ & $97.8 \%$ & $99.6 \%$ \\
\hline \multirow{3}{*}{ 高纬(S) } & 30 & $57.4 \%$ & $88.5 \%$ & $95.9 \%$ & $98.8 \%$ & $99.9 \%$ & $100 \%$ & $100 \%$ \\
\hline & 65 & $47.6 \%$ & $77.4 \%$ & $91.5 \%$ & $97.3 \%$ & $99.7 \%$ & $100 \%$ & $100 \%$ \\
\hline & 90 & $26.7 \%$ & $50.0 \%$ & $68.1 \%$ & $80.8 \%$ & $94.0 \%$ & $98.4 \%$ & $99.6 \%$ \\
\hline
\end{tabular}

偏差随高度的变化有相同的趋势，UMLT高度温度偏 差的标准偏差随高度近似呈指数增长.

(3) 在相同纬度地区和月份下, 同一模式在较低高 度的温度偏差较小, UMLT高度的温度偏差较大. 在北 半球的中高纬度区域, NRLMSISE-00模式的温度偏差 和USSA-76模式的温度偏差在冬夏季的UMLT高度达 到最大．在南半球中高纬度区域，NRLMSISE-00模式 的温度偏差和USSA-76模式的温度偏差均在冬季中间 层顶附近达到最大．在低纬地区，NRLMSISE-00模式 在低热层高度的温度偏差较大; USSA-76模式的温度 偏差在 4 月和 11 月的中间层顶和平流层顶较大.

(4) 同一模式在相同纬度地区和月份下，高度越 高, 温度偏差的标准差越大, 温度偏差分布越离散. 在 两个半球的中高纬度区域，冬季的温度偏差的标准差 大于夏季的温度偏差的标准差. 低纬地区，温度偏差 标准差极值分布的季节特征不明显.

(5) 同一模式, 在高纬地区，均方根误差在夏季中
间层顶附近达到最大．在中纬地区 $70 \mathrm{~km}$ 以下，相同 的高度下，冬季的均方根误差比其他季节的均方根误 差大；在 $70 \mathrm{~km}$ 以上，相同高度下，均方根误差在冬 季和夏季较大. 低纬地区的均方根误差无明显季节 变化.

(6) 同一模式在相同纬度地区和温度偏差要求下, 置信度随着高度的升高而降低；在相同的高度和温度 偏差要求下，置信度随着纬度的升高而降低；除 $90 \mathrm{~km}$ 低纬度和南半球中纬度外, 其他纬度和高度 NRLMSISE-00模式的置信度高于同等条件下USSA76 模式. 在以 $90 \mathrm{~km}$ 为代表的低热层高度, NRLMSISE00 模式在低纬和南半球中纬度地区模式的参数化方案 有待于进一步优化.

大气模式温度和真实大气温度之间的差异存在一 定的时空变化特征, 通过分析两者之间的差异, 为大气 模式在航空航天领域的应用提供参考，也为大气模式 的修正及新模式的研发提供参考依据.

致谢感谢TIMED/SABER工作组提供的探测数据(http://saber.gats-inc.com/data_services.php); 感谢中国科学院国家空 间科学中心空间环境预报中心提供的F10.7 和AP指数. 


\section{参考文献}

1 Kim S I, Rahman H, Hassan I. Effect of turbine inlet temperature on rotor blade tip leakage flow and heat transfer. Int Jnl Num Meth HFF, 2012, 22: 73-93

2 Rahman M H, Kim S I, Hassan I. Effects of inlet temperature uniformity and nonuniformity on the tip leakage flow and rotor blade tip and casing heat transfer characteristics. J Turbomach, 2012, 134: 021001

3 The U. S. Committee on Extension to the Standard Atmosphere (COESA). U. S. Standard Atmosphere, 1976. Washington D C: U. S. Government Printing Office, 1976

4 Justus C G, Jeffries III W R, Yung S P, et al. The NASA/MSFC Global Reference Atmospheric Model-1995 Version (GRAM-95). Technical Report, National Aeronautics and Space Administration, 1995

5 Hedin A E. MSIS-86 Thermospheric Model. J Geophys Res, 1987, 92: 4649-4662

6 Hedin A E. Extension of the MSIS thermosphere model into the middle and lower atmosphere. J Geophys Res, 1991, 96: 1159-1172

7 Chen X X, Hu X, Xiao C Y, et al. Correction method of the low earth orbital neutral density prediction based on the satellites data and NRLMSISE-00 model (in Chinese). Chin J Geophys, 2013, 56: 3246-3254 [陈旭杏, 胡雄, 肖存英, 等. 基于卫星数据和NRLMSISE-00模型的 低轨道大气密度预报修正方法. 地球物理学报, 2013, 56: 3246-3254]

8 Weng L B, Fang H X, Ji C H, et al. Comparison between the CHAMP/STAR derived thermospheric density and the NRLMSISE-00 model (in Chinese). Chin J Space Sci, 2012, 32: 713-719 [翁利斌, 方涵先, 季春华, 等. 基于卫星加速度数据反演的热层大气密度与NRLMSISE-00模 式结果的比较研究. 空间科学学报, 2012, 32: 713-719]

9 Chen L, Liu D, Deng Z X, et al. Accuracy analysis and applicability of the atmosphere model (in Chinese). Chin J Radio Sci, 2014, 29: 774-779 [陈亮, 刘针, 邓忠新, 等. 大气模型的适用性分析及应用研究. 电波科学学报, 2014, 29: 774-779]

10 Picone J M, Hedin A E, Drob D P, et al. NRLMSISE-00 empirical model of the atmosphere: Statistical comparisons and scientific issues. J Geophys Res, 2002, 107: SIA 15-1-SIA 15-16

11 Wang H B, Zhao C Y. Use CHAMP/STAR accelerometer data to evaluate atmospheric density models during solar maximum year (in Chinese). Acta Astron Sin, 2008, 49: 169-178 [汪宏波, 赵长印. 用CHAMP加速仪数据校验太阳活动峰年的大气模型精度. 天文学报, 2008, 49: 169178]

12 Wan T, Liu H W, Fan J. Error band and confidence coefficient of atmospheric density models around altitude $100 \mathrm{~km}$ (in Chinese). Sci Sin-Phys Mech Astron, 2015, 45: 124706 [万田, 刘洪伟, 禁菁. $100 \mathrm{~km}$ 附近大气密度模型的误差带和置信度. 中国科学: 物理学 力学 天文学, 2015, 45: 124706]

13 Xu J Y, Ji Q, Yuan W, et al. Comparisonbetween the TIMED observed global temperature distribution and the NRLMSISE-00 empirical atmospheric model (in Chinese). Chin J Space Sci, 2006, 26: 177-182 [徐寄遥, 纪巧, 袁桦, 等. TIMED卫星探测的全球大气温度分布及其与 经验模式的比较. 空间科学学报, 2006, 26: 177-182]

14 Mertens C J, Russell III J M, Mlynczak M G, et al. Kinetic temperature and carbon dioxide from broadband infrared limb emission measurements taken from the TIMED/SABER instrument. Adv Space Res, 2009, 43: 15-27

15 Cao W X, Zhang S D, Yi F, et al. Variation of the mesopause observed by SABER/TIMED satellite (in Chinese). Chin J Geophys, 2012, 55: 2489-2497 [操文祥, 张绍东, 易帆, 等. 中间层顶变化的 SABER/TIMED卫星观测. 地球物理学报, 2012, 55: 2489-2497]

16 Huang F T, Mayr H G, Reber C A, et al. Diurnal variations of temperature and winds inferred from TIMED and UARS measurements. J Geophys Res, 2006, 111: A10S04

17 Huang F T, McPeters R D, Bhartia P K, et al. Temperature diurnal variations (migrating tides) in the stratosphere and lower mesosphere based on measurements from SABER on TIMED. J Geophys Res, 2010, 115: D16121

18 Zhang X, Forbes J M, Hagan M E, et al. Monthly tidal temperatures 20-120 km from TIMED/SABER. J Geophys Res, 2006, 111: A10S08

19 Wrasse C M, Fechine J, Takahashi H, et al. Temperature comparison between CHAMP radio occultation and TIMED/SABER measurements in the lower stratosphere. Adv Space Res, 2008, 41: 1423-1428

20 Gong X Y, Hu X, Wu X C. et al. Comparison of temperature measurement between COSMIC atmospheric radio occultation and SABER/TIMED (in Chinese). Chin J Geophys, 2013, 56: 2152-2162 [宫晓艳, 胡雄, 吴小成, 等. COSMIC大气掩星与SABER/TIMED探测温度数据比较. 地球 物理学报, 2013, 56: 2152-2162] 
21 Xiao C Y, Hu X, Wang B, et al. Quantitative studies on the variations of near space atmospheric fluctuation (in Chinese). Chin J Geophys, 2016, 59: 1211-1221 [肖存英, 胡雄, 王博, 等. 临近空间大气扰动变化特性的定量研究. 地球物理学报, 2016, 59: 1211-1221]

22 Xiao C, Hu X, Tian J. Global temperature stationary planetary waves extending from 20 to $120 \mathrm{~km}$ observed by TIMED/SABER. J Geophys Res, 2009, 114: D17101

23 Chen Z Y, Lv D R. Seasonal variations of the MLT tides in $120^{\circ}$ E meridian (in Chinese). Chin J Geophys, 2007, 50: 691-700 [陈泽宇, 吕达仁. 东经 $120^{\circ} \mathrm{E}$ 中间层和低热层大气潮汐及其季节变化特征. 地球物理学报, 2007, 50: 691-700]

24 Chen Z Y, Lv D R. Satellite remote sensing of the characteristics of MLT mean temperatures in the $120^{\circ} \mathrm{E}$ meridian: The mesopause (in Chinese). Chin J Geophys, 2008, 51: 982-990 [陈泽宇, 吕达仁. 卫星遥感东经 $120^{\circ}$ 子午圈MLT典型温度结构中间层顶统计分析. 地球物理学报, 2008, 51: 982-990]

25 Yue C, Yang G, Wang J, et al. Lidar observations of the middle atmospheric thermal structure over north China and comparisons with TIMED/ SABER. J Atmos Sol-Terrestrial Phys, 2014, 120: 80-87

26 Fechine J, Wrasse C M, Takahashi H, et al. Lower-mesospheric inversion layers over brazilian equatorial region using TIMED/SABER temperature profiles. Adv Space Res, 2008, 41: 1447-1453

27 Xiao C Y, Hu X, Yang J F, et al. Studies on the characteristics of atmospheric density at $38^{\circ} \mathrm{N}$ in nearspace and its modeling technique (in Chinese). J Beijing Univ Aeronaut Astron, 2016, doi: 10.13700/j.bh.1001-5965.2016.0735 [肖存英, 胡雄, 杨钧峰, 等. 临近空间 $38^{\circ} \mathrm{N}$ 大气密度 特性及建模技术研究. 北京航空航天大学学报, doi: 10.13700/j.bh.1001-5965.2016.0735]

28 Offermann D, Jarisch M, Oberheide J, et al. Global wave activity from upper stratosphere to lower thermosphere: A new turbopause concept. J Atmos Sol-Terrestrial Phys, 2006, 68: 1709-1729

29 Offermann D, Gusev O, Donner M, et al. Relative intensities of middle atmosphere waves. J Geophys Res, 2009, 114: 605-617

30 Yi F, Klostermeyer J, Ruester R. VHF radar observation of gravity wave critical layers in the mid-latitude summer mesopause region. Geophys Res Lett, 1991, 18: 697-700

31 Dong L W. Mesospheric temperature inversion layers: Recent observations from UARS ISAMS and MLS. Recent Res Devel Geophys, 1999, 3: $37-44$

32 Williams B P. Gravity waves in the arctic mesosphere during the MaCWAVE/MIDAS summer rocket program. Geophys Res Lett, 2004, 31: L24S05 


\title{
Evaluation of atmospheric empirical model based on TIMED/ SABER satellite temperature data
}

\author{
CHENG Xuan ${ }^{1,2}$, XIAO CunYing $^{1 *}$, HU Xiong $^{1}$ \& YANG JunFeng ${ }^{1}$ \\ ${ }^{1}$ Laboratory of Near Space Environment, National Space Science Center, Chinese Academy of Sciences, Beijing 100190, China; \\ ${ }^{2}$ University of Chinese Academy of Sciences, Beijing 100049, China
}

\begin{abstract}
A total of 15 years of global temperature data from 2002-2016 as measured by TIMED/SABER were taken as standard to compare with those of USSA-76 and NRLMSISE-00. The accuracy of the two atmospheric models was analyzed by calculating daily mean and monthly mean temperature deviations and standard deviations of temperature deviations at different altitudes. The confidence of the model under different temperature deviations is calculated through setting the deviation between the models and the satellite observation temperature. The results show that the temperature data calculated by the two atmospheric models are in good agreement with the temperature observed by satellite. The daily mean and monthly mean accuracy of the NRLMSISE-00 are higher than those of the USSA-76. In the UMLT region, the two atmospheric models have yet to be improved to characterize the true atmosphere at the mid-high latitudes of upper mesosphere in summer and winter. The overall ability of the NRLMSISE-00 to represent the true atmosphere is superior to the USSA-76. In addition, the confidence decreases with the increase of altitude in the same latitude area and temperature deviation requirements. The confidence decreases with increasing latitude in the same altitude and temperature deviation requirements. By analyzing the differences between the atmospheric models and satellite observations. This paper provides a reference for the application of atmospheric models in engineering and provides the basis for the subsequent correction of atmospheric modes.
\end{abstract}

atmospheric model, temperature, TIMED/SABER, NRLMSISE-00, USSA-76, evaluation

PACS: 43.28.Vd, 46.15.-x, 96.12.Jt, 92.60.hv

doi: $10.1360 /$ SSPMA2018-00091 